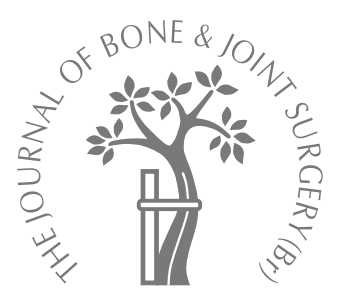

B. Magnan,

R. Bortolazzi,

A. Marangon,

M. Marino,

C. Dall'Oca,

P. Bartolozzi

From the Department of Orthopaedics, University of Verona, Verona, Italy

B. Magnan, MD, Orthopaedic Surgeon

R. Bortolazzi, MD,

Orthopaedic Surgeon

A. Marangon, MD,

Orthopaedic Surgeon

M. Marino, MD, Orthopaedic

Surgeon

III C. Dall'Oca, MD, Orthopaedic Surgeon

P. Bartolozzi, MD, Professor Department of Orthopaedics University of Verona,

Policlinico "G. B. Rossi",

Piazzale Lodonco Amtomio

Scuro 10, 37134 Verona, Italy.

Correspondence should be sent to Dr B. Magnan; e-mail: brunomagnan@virgilio.it

(C)2006 British Editorial Society of Bone and Joint Surgery doi:10.1302/0301-620X.88B11. $17759 \$ 2.00$

$J$ Bone Joint Surg [Br] 2006:88-B:1474-9.

Received 10 February 2006; Accepted after revision 21 June 2006

\title{
External fixation for displaced intra-articular fractures of the calcaneum
}

\begin{abstract}
A minimally-invasive procedure using percutaneous reduction and external fixation can be carried out for Sanders' type II, III and IV fractures of the os calcis. We have treated 54 consecutive closed displaced fractures of the calcaneum involving the articular surface in $\mathbf{5 2}$ patients with the Orthofix Calcaneal Mini-Fixator. Patients were followed up for a mean of 49 months (27 to 94) and assessed clinically with the Maryland Foot Score and radiologically with radiographs and CT scans, evaluated according to the Score Analysis of Verona. The clinical results at follow-up were excellent or good in 49 cases $(\mathbf{9 0 . 7 \% )}$, fair in two $(3.7 \%)$ and poor in three $(5.6 \%)$. The mean pre-operative Böhler's angle was $6.98^{\circ}\left(5.95^{\circ}\right.$ to $\left.19.86^{\circ}\right)$, whereas after surgery the mean value was $21.94^{\circ}\left(12.58^{\circ}\right.$ to $\left.31.30^{\circ}\right)(p<0.01)$. Excellent results on CT scanning were demonstrated in 24 cases $(44.4 \%)$, good in 25 $(46.3 \%)$, fair in three $(5.6 \%)$ and poor in two $(3.7 \%)$. Transient local osteoporosis was observed in ten patients $(18.5 \%)$, superficial pin track infection in three $(5.6 \%)$, and three patients $(5.6 \%)$ showed thalamic displacement following unadvised early weight-bearing.

The clinical results appear to be comparable with those obtainable with open reduction and internal fixation, with the advantages of reduced risk using a minimally-invasive technique.
\end{abstract}

Calcaneal fractures involving the joints are disabling injuries which can jeopardise work and day-to-day activities. ${ }^{1}$ Such injuries represent more than $2 \%$ of all fractures, and $65 \%$ to $70 \%$ of them have displacement of the articular surfaces. ${ }^{2}$ The anatomy and biomechanics of the hindfoot account for the difficulties in treating this type of fracture because the calcaneum is constantly subjected to compression forces, provides a lever-arm in the Achillescalcaneal-plantar system, and has articular surfaces which form two complex joints, the mid tarsal (Chopart) and the subtalar. Many authors ${ }^{3-9}$ have attempted to define algorithms for conservative and surgical treatment depending on articular involvement and the degree of displacement. Despite several comparative studies between conservative and surgical methods, ${ }^{3,10}$ the management of intraarticular calcaneal fractures remains controversial. There are a number of reasons for this; 1) difficulty in obtaining reduction using conservative methods, often leading to pain, hindfoot deformity, impingement and disturbed gait; 2) difficulty of reduction and fixation, even with an open surgical approach; 3) the high risk of major complications related to open surgery: up to a $16 \%{ }^{10}$ incidence of wound complications ${ }^{11-15}$ and a $20 \%{ }^{12-14}$ incidence of infection in closed fractures, increasing to $33 \%{ }^{12}$ in open injuries. The risk of nerve injury is $1.6 \% .^{11}$

Although open reduction and internal fixation is currently considered the treatment of choice for fractures type II, III and IV, according to Sander's classification, ${ }^{13,16}$ uncertainty remains about the comparative final results of surgical and conservative treatment, ${ }^{3,7,9,10}$ as neither provides good results without the risk of considerable early and delayed complications.

External fixation with closed or percutaneous reduction, following a pre-operative CT scan, is a minimally-invasive technique that can obtain reduction and stable fixation of most displaced articular fractures while greatly reducing the risks of open surgery. There are many preliminary studies ${ }^{17-20}$ on the use of external fixation in calcaneal fractures, and this method is generally accepted for salvage procedures or open fractures. $^{21}$

The results described in the most recent studies are better than those observed in the past, and are now comparable to treatment by open reduction and internal fixation (ORIF). ${ }^{19}$ This may be related to early mobilisation of the 


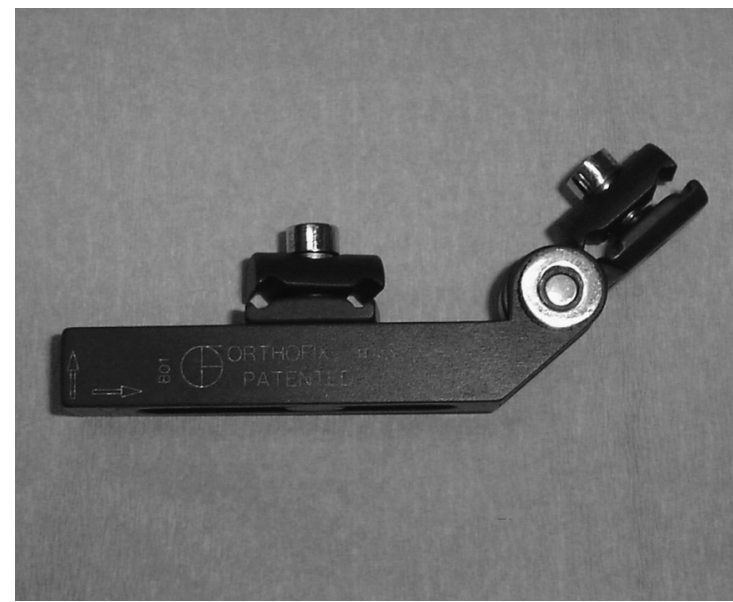

Fig. 1a

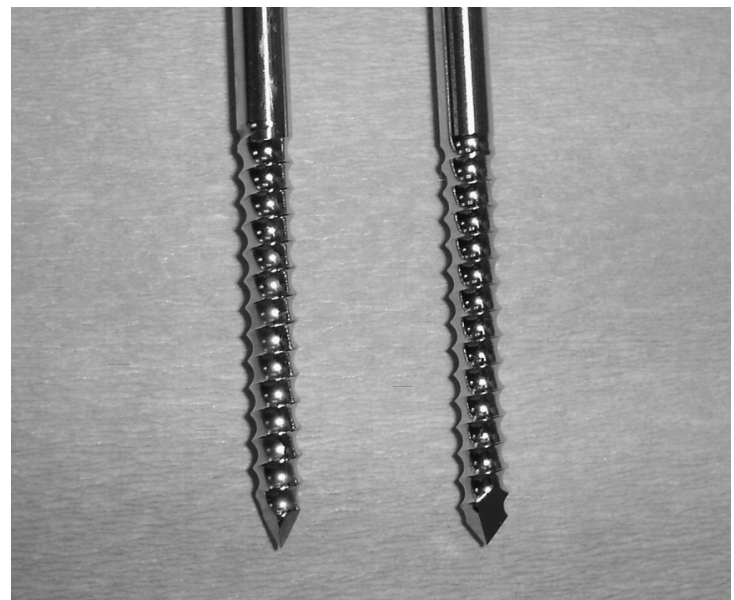

Fig. 1b

Figure 1a - The Orthofix Heel Mini-Fixator (Orthofix spa, Verona, Italy). Figure 1b - Self-drilling and self-threading 4 mm diameter screws.

peritalar joints thanks to the stability provided by external fixation devices. However, there are no reports with longterm results of the use of closed or percutaneous reduction and external fixation in displaced articular fractures. This was the aim of this study.

\section{Patients and Methods}

Between 1996 and 2002, 54 consecutive closed articular displaced calcaneal fractures in 52 patients were treated with percutaneous reduction and external fixation. There were 38 men and 14 women, with a mean age of 48.6 years (20 to 76$)$. A total of 15 fractures $(28 \%)$ were classified as Sanders type II, $31(57 \%)$ as type III and $8(15 \%)$ as type IV.

We considered that external fixation was indicated for the treatment of Sanders' type II, III and IV fractures. External fixation was not indicated for two fractures (not included in this series) with large cranial displacement of the lateral wall, in which open reduction of the subtalar joint was mandatory. The procedure may be employed in patients with diabetes mellitus or peripheral vascular disease. Osteoporosis was not regarded as a contraindication.

Surgery was performed at a mean of 5.4 days (1 to 14 ) after trauma. Minimally-invasive percutaneous external fixation allowed early treatment because swelling and poor soft-tissue injury did not represent an absolute contraindication to operation.

Surgical technique. The operation is performed under image intensifier control and external fixation obtained using a device from the Orthofix Mini-Fixator series (Orthofix spa, Verona, Italy, series M300) of appropriate size. These are equipped with a uniplanar joint (Fig. 1a), perpendicular axis pins, and a small slide supporting one of the clamps which enables the frame to be lengthened or shortened. The pins are self-drilling and self-tapping, with a diameter of $4 \mathrm{~mm}$ (Fig. 1b), and are available in two lengths, $70 \mathrm{~mm}$ and $90 \mathrm{~mm}$.

The patient is placed in the lateral decubitus position, exposing the lateral surface of the heel. The first step consists of percutaneous reduction of the thalamic articular fragments through a $1 \mathrm{~cm}$ lateral incision over the sinus tarsi. Using a small lever inserted below the fragments from the floor of the sinus (Fig. 2a), the articular surface is raised and rotated until it is fully reduced. Fixation is then performed. Pin sites are selected in relation to the fracture pattern. The first two are placed in the thalamic fragments (Fig. 2b), and supported by a counterthrust exerted by two other pins positioned according to the fracture pattern: on the posterior apophysis in 'joint depression'-type fractures (Essex-Lopresti ${ }^{22}$ ) in 24 cases $(44.5 \%)$, on the anterior tuberosity in 'tongue'-type fractures with rotation of the thalamic surface in $21(38.9 \%)$, and in both in 'burst' fractures in seven cases $(12.9 \%)$. Where there is severe comminution of the anterior tuberosity, as occurred in two cases, one or both pins may be positioned in the lateral aspect of the cuboid.

The body of the mini-fixator serves as a template for positioning the other pairs of pins (Fig. 2c). When the clamps are tightened, the inclination of the pins in the posterior tuberosity may permit correction of varus and valgus deformity of the body of the calcaneum relative to the articular surface by up to approximately $20^{\circ}$.

After the fixator has been applied, further reduction and elevation of the thalamic fragment may be carried out by elongating the clamps on the slide using the appropriate key in the distraction mechanism (Figs $2 \mathrm{~d}$ and 2e). A soft dressing is then applied over the fixator.

Post-operatively, weight-bearing is not permitted for eight weeks, and the dressing is renewed every week. Passive and active mobilisation of the ankle and peritalar joints 


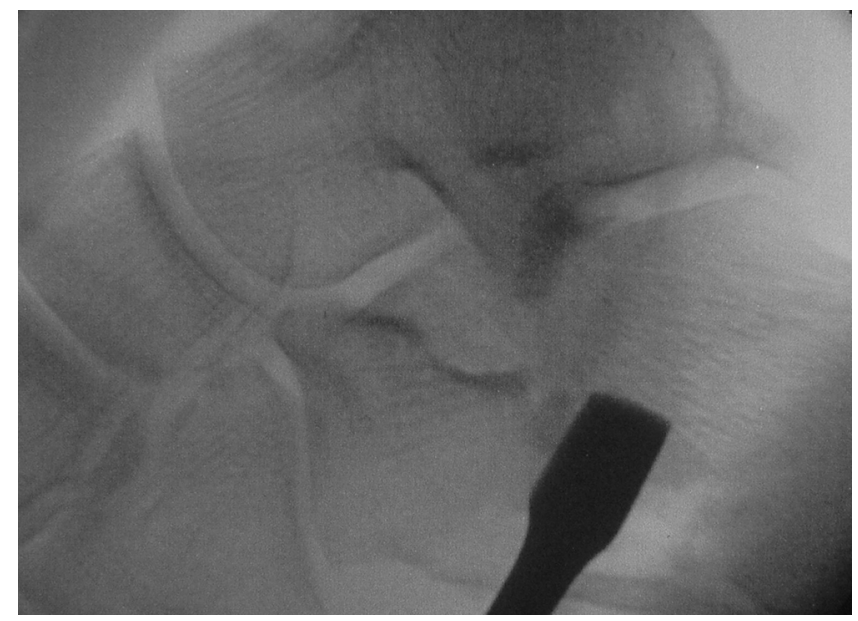

Fig. 2a

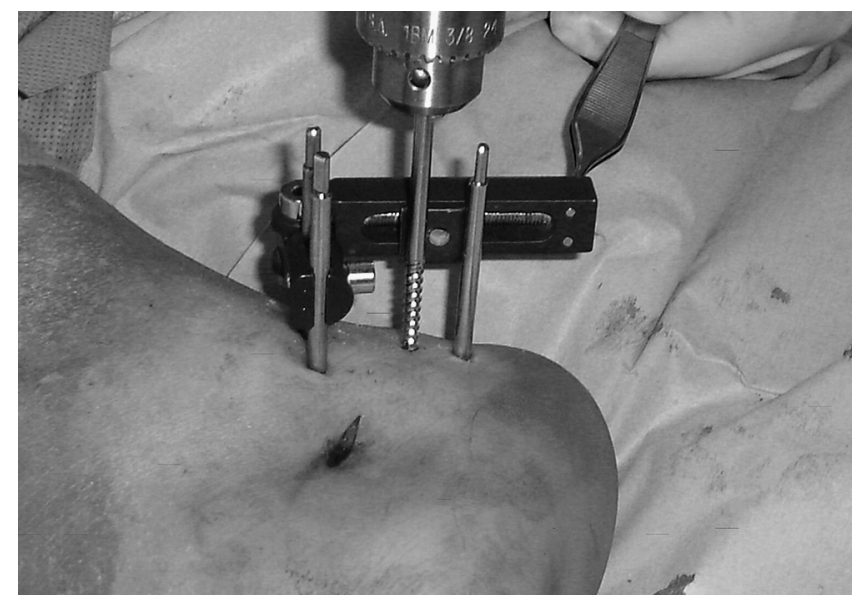

Fig. $2 c$
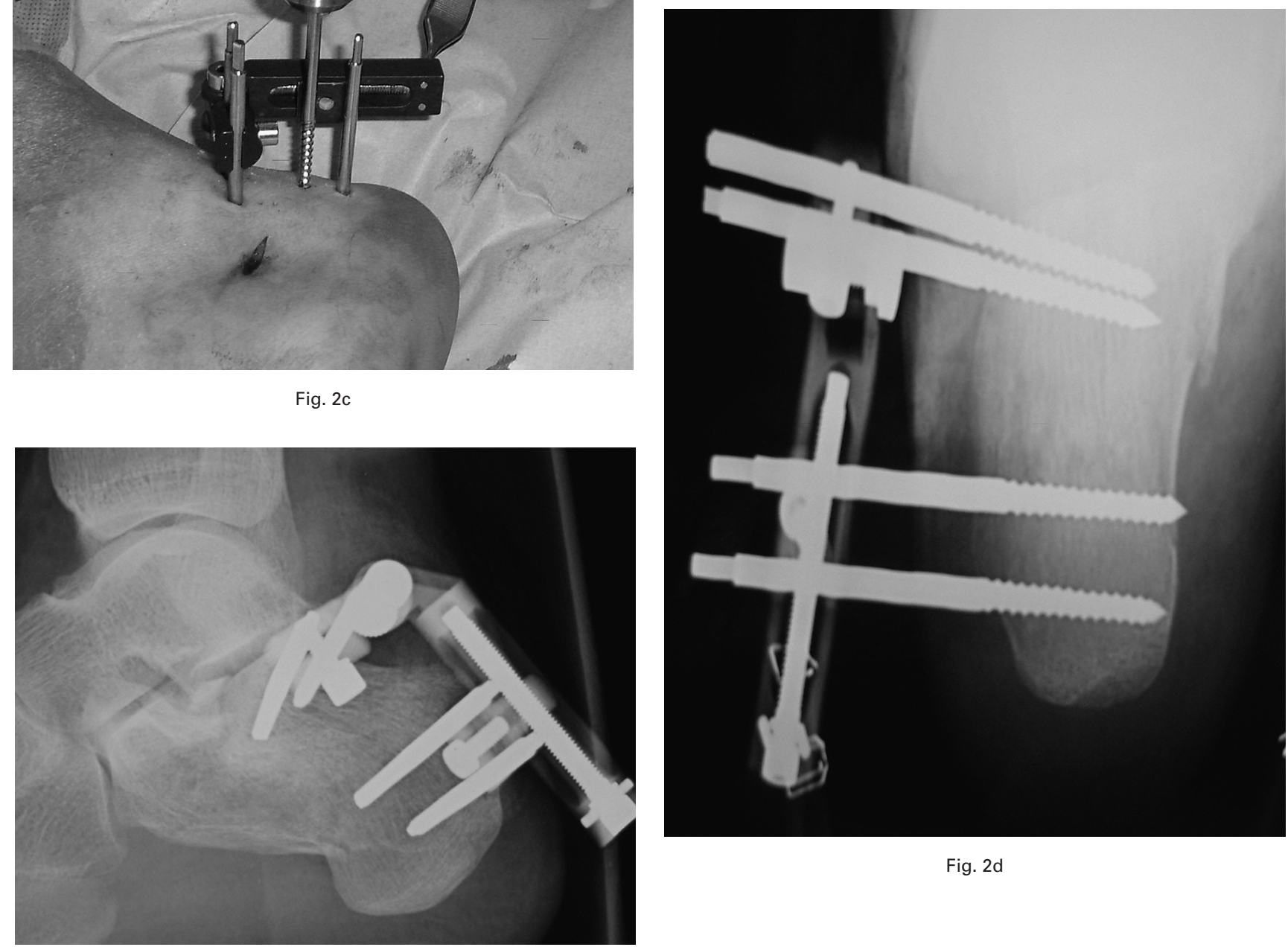

Fig. 2d

Fig. $2 \mathrm{e}$

Intra-operative and fluoroscopic images of the surgical technique showing a) percutaneous reduction of the articular fragment, b) the first two pins placed in the thalamic fragments, c) the body of the mini-fixator serving as a template for positioning the other pairs of pins, and d) and e) post-operative anteroposterior and lateral radiographs. 
is begun immediately. Radiological examination is undertaken at four, eight and 12 weeks. The fixator is removed after eight weeks in Sanders' type II fractures, and after ten weeks in type III and type IV.

When the fixator is removed, intensive/active and passive mobilisation is continued for two more months. Patients are encouraged to use orthotic shoes during the nonweight-bearing period and while walking with the fixator in place. After removal of the device, normal sport shoes are permitted.

Assessment. The Maryland foot score ${ }^{13,23}$ was used for clinical assessment at follow-up. This is a validated ${ }^{24}$ scor- $^{-}$ ing system ranging from a minimum of 0 to a maximum of 100 (excellent, 90 to 100; good, 75 to 89; fair, 50 to 74; failure $<50)$. It evaluates subjective and objective elements such as pain (maximum score 45), function (maximum score 40, subdivided into gait, stability, use of walking aids, limp, type of shoes required, walking distance), cosmesis (maximum score 10), and movement of the ankle, subtalar, midfoot and metatarsophalangeal joints (maximum score 5 ). As suggested by Sanders et al, ${ }^{13}$ we measured the range of movement of the subtalar joint according to the method of Morrey and Wiedeman. ${ }^{25}$ Pre-operative anteroposterior, lateral and tangential radiographs measuring the Böhler's angle $^{26}$ and CT scans of the calcaneum were taken of all fractures treated in this study.

CT images were used to classify the type of fracture according to the Sanders' classification and the Score analysis of Verona (SAVE), ${ }^{26}$ which correlates CT images and functional outcome. Five CT parameters are considered: vertical alignment (varus deformity: 3 points, valgus: 4, normal: 7), longitudinal alignment (varus deformity: 3 points, valgus: 4; normal: 7), calcaneal height (severe reduction: 3 points, slight reduction: 5, normal: 7), position of the sustentaculum tali (severe dislocation: 1 point, slight dislocation: 2, normal: 5), and congruity of the subtalar joint (poor: 0 points, fair: 2, good: 4)..$^{13,18,27-29}$ A score ranging between 26 and 30 is considered excellent, 20 and 25 good, 15 and 19 fair, and lower than 15 failure. The score showed statistical correlation with the clinical outcome as judged by the Maryland Foot Score in the original study of Magnan et al. ${ }^{27}$ Patients were assessed, with standard radiographs every four weeks for three months, and a CT scan at the final follow-up.

Statistical analysis. The statistical significance of the changes in radiological values of Böhler's angle, ${ }^{26}$ and the CT SAVE score was determined using Student's $t$-test, with values below 0.05 being considered significant.

\section{Results}

All 52 patients were followed for a mean of 49 months (27 to 94$)$. None required further surgery.

Clinical results were assessed at follow-up, according to the Maryland foot score, being excellent in 26 of 54 cases $(48.1 \%)$ (Fig. 3), good in 23 (42.6\%), fair in two $(3.7 \%)$ and poor in three $(5.6 \%)$ (Table I). The three poor results
Table I. Clinical results of the 54 fractures (\%) according to the Maryland foot score at the time of final follow-up

\begin{tabular}{lrr}
\hline Maryland foot score & & \\
\hline Excellent & 26 & $(48.1)$ \\
Good & 23 & $(42.6)$ \\
Fair & 2 & $(3.7)$ \\
Poor & 3 & $(5.6)$ \\
\hline
\end{tabular}

Table II. CT evaluation of the 54 fractures $(\%)$ according to the score analysis of Verona (SAVE) score at the final follow-up

\begin{tabular}{lrr}
\hline SAVE score & & \\
\hline Excellent & 24 & $(44.4)$ \\
Good & 25 & $(46.3)$ \\
Fair & 3 & $(5.6)$ \\
Poor & 2 & $(3.7)$ \\
\hline
\end{tabular}

were observed in non-compliant patients in whom collapse of the calcaneal body was caused by early weight-bearing against medical instruction. At follow-up we evaluated the CT scans of treated heels according to the SAVE score, which was excellent in 24 of 54 cases (44.4\%), good in 25 $(46.3 \%)$, fair in three $(5.6 \%)$ and poor in two $(3.7 \%)$ (Table II). The average pre-operative SAVE score was 17.58 (12.60 to 22.56), and the mean post-operative value at follow-up was 25.66 (23.33 to 28.1) (p<0.01).

The mean pre-operative Böhler's angle 26,30 was $6.98^{\circ}$ $\left(5.95^{\circ}\right.$ to $\left.19.86^{\circ}\right)$ and the mean post-operative value was $21.94^{\circ}\left(12.58^{\circ}\right.$ to $\left.31.30^{\circ}\right)(\mathrm{p}<0.01)$. In 39 cases $(72.2 \%)$ the Böhler's angle was improved by $20^{\circ}$.

Regarding complications, we observed transient local post-traumatic osteoporosis, which resolved within four months, in ten of 54 fractures $(18.5 \%)$. There were three cases of superficial pin track infection $(5.6 \%)$. These were treated with local dressing and oral antibiotics for two weeks without requiring early removal of the pins. The three patients $(5.6 \%)$ with thalamic displacement following early weight-bearing did not receive further surgery.

\section{Discussion}

The main goal of treatment for displaced fractures of the calcaneum should be the restoration of the three-dimensional structure, with emphasis on correct alignment in the coronal and axial planes and the height of the calcaneal body, ${ }^{8,9,13,27,28}$ rather than anatomical reconstruction of the congruency of the subtalar articular fragments. ${ }^{17,19,20,27,28}$ The use of an external fixator appears to achieve this aim. The reduction is monitored by a stable fixation and the risk of major complications is reduced.

Anatomical restoration of the subtalar joint facet is very difficult to achieve, particularly with percutaneous reduction and external fixation using two single pins to stabilise 


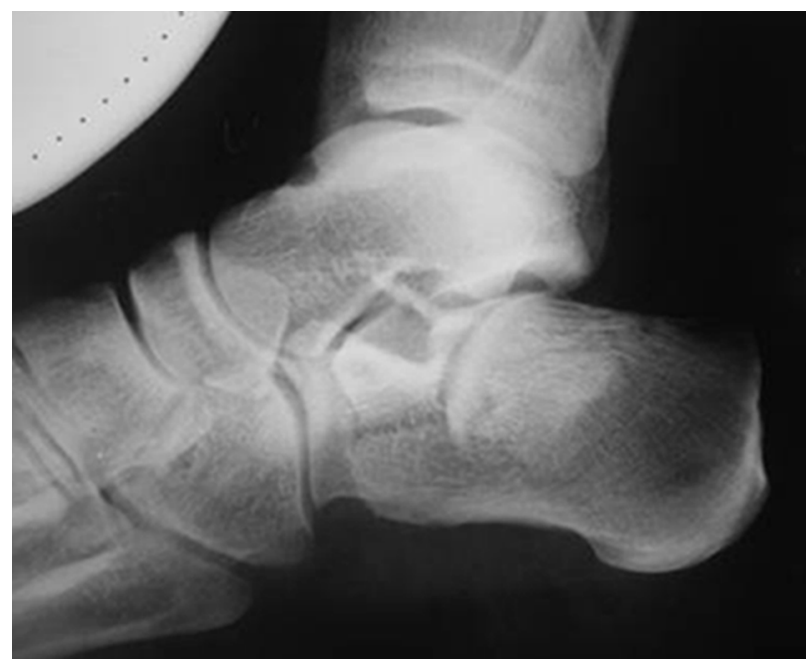

Fig. 3a

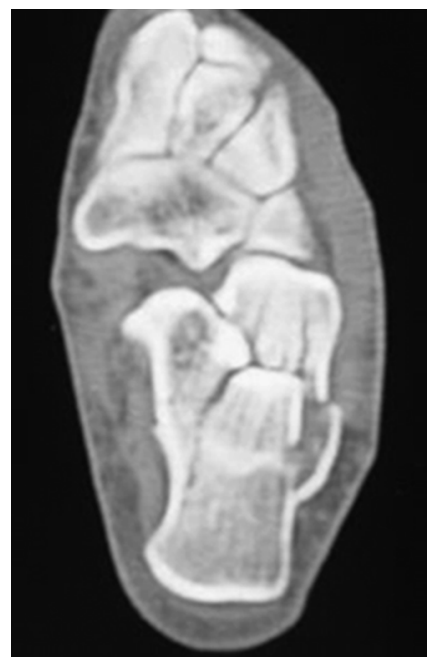

Fig. $3 b$

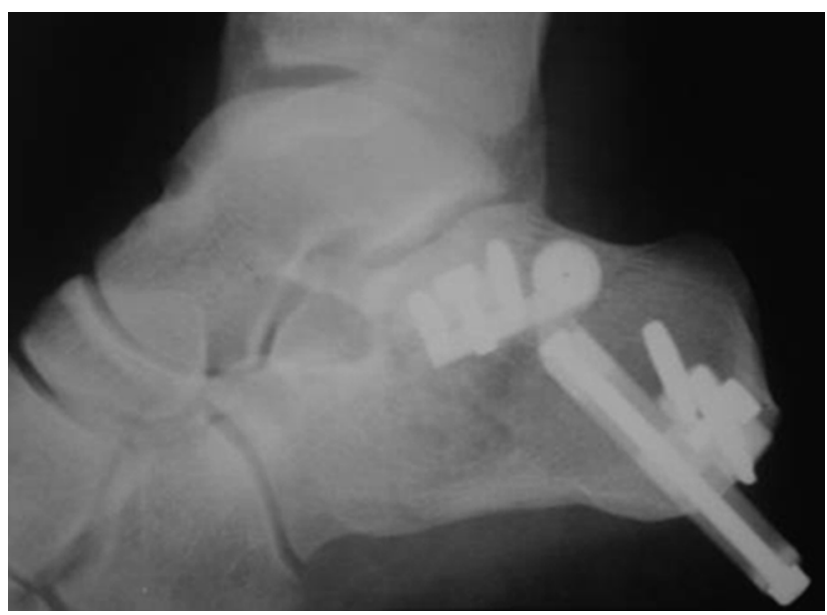

Fig. 3c

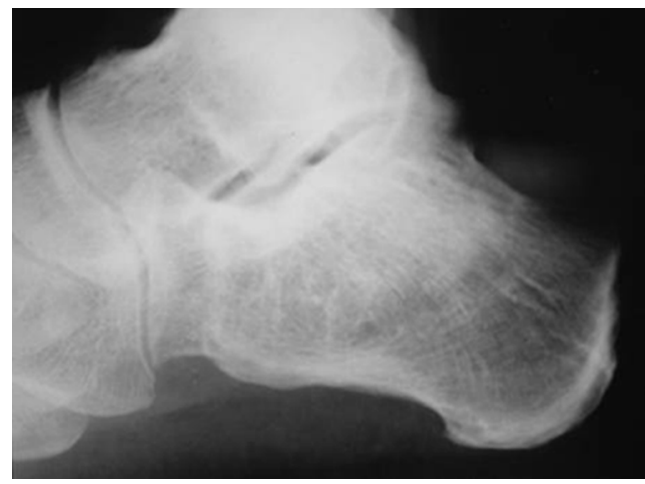

Fig. $3 d$

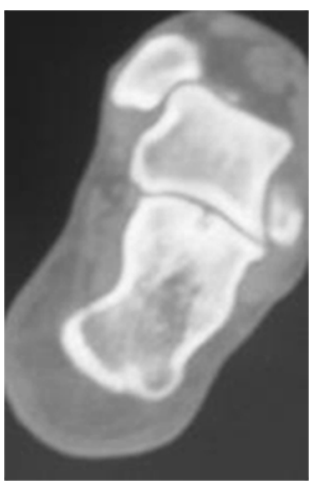

Fig. 3e

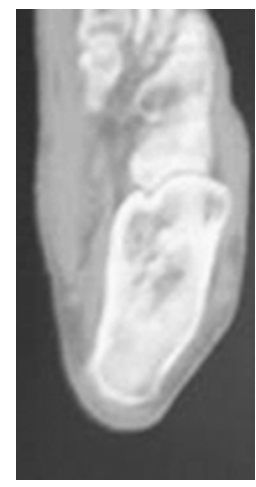

Fig. $3 f$

A 42-year-old male with a Sanders' type II fracture treated with percutaneous reduction and external fixation showing a) pre-operative lateral radiograph, b) pre-operative axial CT scan, c) post-operative lateral radiograph, d) lateral radiograph at follow-up, e) coronal and f) axial CT scans at 3 years follow-up. 
the articular fragments, as demonstrated by CT evaluation at follow-up. Some degree of stiffness and degenerative arthritis of the subtalar joint following a displaced articular fracture is usually unavoidable whatever the chosen treatment, ${ }^{23}$ owing to severe damage to the articular cartilage which almost always sustains high-energy axial load. ${ }^{31}$ The results of our series indicate that neither the subtalar mobility nor the clinical outcome seem to be significantly affected by obtaining congruency of the subtalar facet when compared with the results reported with open forms of treatment which specifically aim to reduce the articular fragments anatomically, 3,19 but correlate more with the results following early post-operative mobilisation and the restoration of Böhler's angle, according to Paul et al. ${ }^{9}$

In order to reduce the specific complications related to the external fixation, such as loose screws, infection and loss of reduction, it is important to monitor patients strictly, with weekly observations until the fixator is removed. In our series this regimen prevented early removal of the fixator prior to consolidation of the fracture.

Percutaneous reduction and external fixation proved to be a reliable technique for obtaining stable reconstruction of fractures of the os calcis. The clinical results appear to be comparable to those observed following open reduction and internal fixation, ${ }^{13,14,23,32-35}$ and are impressive compared with those of Sanders et $\mathrm{al}^{13}$ and Heffernan et al. ${ }^{24}$ They are similar to a small series of calcaneal fractures treated by external fixation reported by Talarico et al, ${ }^{19}$ who obtained $92 \%$ of excellent and good outcomes using the Maryland foot score. The added advantages of minimally-invasive procedures are the considerably shortened operating time and hospital stay, and the reduced risk of complications related to surgical exposure.

We therefore consider that external fixation of displaced intra-articular calcaneal fractures is a valid alternative treatment compared with open reduction and internal fixation.

No benefits in any form have been received or will be received from a commercial party related directly or indirectly to the subject of this article.

\section{References}

1. Coughlin MJ. Calcaneal fractures in industrial patients. Foot Ankle Int 2000;21:896-905.

2. Sanders R, Hansen ST Jr, McReynolds IS. Trauma to the calcaneus and its tendon: fractures of the calcaneous. In: Jahss MH, ed. Disorders of the foot and ankle. Second ed. Philadelphia etc: W. B. Saunders Co., 1991:2326-54.

3. Randle JA, Kreder HJ, Stephen D, et al. Should calcaneal fractures be treated surgically?: a meta-analysis. Clin Orthop 2000;377:217-27.

4. Rammelt S, Zwipp H. Calcaneus fractures: facts, controversies and recent developments. Injury 2004;35:443-61.

5. Thornes BS, Collins AL, Timlin M, Corrigan J. Outcome of calcaneal fractures treated operatively and non-operatively: the effect of litigation on outcomes. Ir J Med Sci 2002;3:155-7.

6. Dooley P, Buckley R, Tough S, et al. Bilateral calcaneal fractures: operative versus nonoperative treatment. Foot Ankle Int 2004;25:47-52.

7. Parmar HV, Triffitt PD, Gregg PJ. Intra-articular fractures of the calcaneum treated operatively or conservatively: a prospective study. J Bone Joint Surg [Br] 1993:75-B:932-7.
8. Eastwood DM, Langkamer VG, Atkins RM. Intra-articular fractures of the calcaneum. Part II: open reduction and internal fixation by the extended lateral transcalcaneal approach. J Bone Joint Surg [Br] 1993;75-B:189-95.

9. Paul M, Peter R, Hoffmeyer P. Fractures of the calcaneum: a review of 70 patients. J Bone Joint Surg [Br] 2004;86-B:1142-5

10. Bajammal S, Tornetta P 3rd, Sanders D, Bhandari M. Displaced intra-articular calcaneal fractures. J Orthop Trauma 2005;19:360-4.

11. Howard JL, Buckley R, McCormack R, et al. Complications following management of displaced intra-articular calcaneal fractures: a prospective randomized trial comparing open reduction internal fixation with nonoperative management. J Orthop Trauma 2003:4:241-9.

12. Benirschke SK, Kramer PA. Wound healing complications in closed and open calcaneal fractures. J Orthop Trauma 2004;18:1-6.

13. Sanders R, Fortin P, DiPasquale T, Walling A. Operative treatment in 120 dis placed intraarticular calcaneal fractures: results using a prognostic computed tomography scan classification. Clin Orthop 1993;290:87-95.

14. Zwipp H, Tscherne H, Thermann H, Weber T. Osteosynthesis of displaced intraarticular fractures of the calcaneus: results in 123 cases. Clin Orthop 1993;290:76-86.

15. Lim EV, Leung JP. Complications of intraarticular calcanear fractures. Clin Orthop 2001;391:7-16.

16. Rajkumar P, Henderson AA. Surgical treatment of displaced intra-articular fractures of the os-calcis. Foot Ankle Surg 2003;9:3-6.

17. Nogarin L, Magnan B, Bragantini A, et al. Trattamento a cielo chiuso degli affossamenti talamici con mini-fissatori esterni. In: Mori F, Gianni S, Traina GC, Massari L, eds. Fratture del calcagno. Progressi in Medicina e Chirurgia del Piede. Vol 3. Bologna: Aulo Gaggi Editore 1994:153-9.

18. Magnan B, Caudana R, Campacci A, et al. Follow-up clinico e radiografico mediante T.C. delle fratture di calcagno trattate con mini FEA. Chir del piede 1992;16:145-50.

19. Talarico LM, Vito GR, Zyryanov SY. Management of displaced intraarticular displaced fractures by using external fixation, minimally invasive open reduction, and early weight-bearing. J Foot Ankle Surg 2004;43:43-50.

20. Paley D, Fischgrund J. Open reduction and circular external fixation of intraarticular calcaneal fractures. Clin Orthop 1993;290:125-31.

21. Kenwright J. Fractures of the calcaneum. J Bone Joint Surg [Br] 1993;75-B:176-

22. Essex-Lopreseti P. The mechanism, reduction, technique and results in fractures of the os calcis. Br J Surg 1952;39:395-419.

23. Sanders R. Displaced intra-articular fractures of the calcaneus. J Bone Joint Surg [Am] 2000;82-A:225-50.

24. Heffernan G, Khan F, Awan N, Riordain CO, Corrigan J. A comparison of outcome scores in os calcis fractures. Ir J Med Sci 2000;169:127-8.

25. Morrey BF, Wiedeman GP Jr. Complications and long-term results of ankle arthrodesis following trauma. J Bone Joint Surg [Am] 1980;62-A:777-84.

26. Böhler L. Diagnosis, pathology and treatment of fractures of the os calcis. $J$ Bone Joint Surg 1931;13:75-83.

27. Magnan B, Montanari M, Brangantini A, Bartolozzi P. A system of prognostic evaluation of CT imaging of heel fractures: the Score Analysis Verona (SAVE) Foot Disease 1995;1:19-25.

28. Richardson ML, Van Vu M, Vincent LM, Sangeorzan BJ, Benirschke SK. CT measurement of the calcaneal varus angle in the normal and fractured hindfoot. J Comput Assist Tomogr 1992;16:261-4.

29. Rosenberg ZS, Feldman F, Singson RD. Intra-articular calcaneal fractures: computed tomographic analysis. Skeletal Radiol 1987;16:105-13.

30. Loucks C, Buckley R. Bohler's angle: correlation with outcome in displaced intra-articular calcaneal fractures. J Orthop Trauma 1999;13:554-8.

31. Borelli J Jr, Torzilli P, Grigiene R, Helfet DL. Effect of impact load on articular cartilage: development of an intra-articular fracture model. J Orthop Trauma 1997:11:319-26.

32. Paley D, Hall H. Intra-articular fractures of the calcaneus: a critical analysis of results and prognostic factors. J Bone Joint Surg [Am] 1993;75-A:342-54.

33. Aldridge JM 3rd, Easley M, Ninley JA. Open calcaneal fractures: results of operative treatment. J Orthop Trauma 2004;18:7-11.

34. Myerson M, Quill GE Jr. Late complications of fracture of the calcaneus. J Bone Joint Surg [Am] 1993;75-A:331-41.

35. Csizy M, Buckley R, Tough S, et al. Displaced intra-articular calcaneal fractures: variables predicting late subtalar fusion. J Orthop Trauma 2003;17:106-12. 